\title{
A potential target gene for the host-directed therapy of mycobacterial infection in murine macrophages
}

\author{
ZHANG BAO ${ }^{1}$, RAN CHEN $^{2,3^{*}}$, PEI ZHANG $^{1}$, SHAN LU $^{1}$, XING CHEN $^{1}$, YAKE YAO $^{1}$, \\ XIAOZHENG JIN ${ }^{2}$, YILAN SUN ${ }^{1}$ and JIANYING ZHOU ${ }^{1 *}$ \\ ${ }^{1}$ Department of Respiratory Medicine, The First Affiliated Hospital, Zhejiang University, Hangzhou, Zhejiang 310003; \\ ${ }^{2}$ Zhejiang JFK Biological Technology Co., Ltd., Hangzhou, Zhejiang 310052; \\ ${ }^{3}$ School of Life Science, Fudan University, Shanghai 200433, P.R. China
}

Received March 24, 2016; Accepted July 6, 2016

DOI: $10.3892 / \mathrm{ijmm} .2016 .2675$

\begin{abstract}
Mycobacterium tuberculosis (MTB), one of the major bacterial pathogens for lethal infectious diseases, is capable of surviving within the phagosomes of host alveolar macrophages; therefore, host genetic variations may alter the susceptibility to MTB. In this study, to identify host genes exploited by MTB during infection, genes were non-selectively inactivated using lentivirus-based antisense RNA methods in RAW264.7 macrophages, and the cells that survived virulent MTB infection were then screened. Following DNA sequencing of the surviving cell clones, 26 host genes affecting susceptibility to MTB were identified and their pathways were analyzed by bioinformatics analysis. In total, 9 of these genes were confirmed as positive regulators of collagen $\alpha-5$ (IV) chain (Col4a5) expression, a gene encoding a type IV collagen subunit present on the cell surface. The knockdown of Col4a5 consistently suppressed intracellular mycobacterial viability, promoting the survival of RAW264.7 macrophages following mycobacterial infection. Furthermore, Col4a5 deficiency lowered the $\mathrm{pH}$ levels of intracellular vesicles, including endosomes, lysosomes and phagosomes in the RAW264.7 cells. Finally, the knockdown of Col4a5 post-translationally increased microsomal vacuolar-type $\mathrm{H}^{+}$-ATPase activity in macrophages, leading to the acidification of intracellular vesicles. Our findings reveal a novel role for $\mathrm{Col} 4 \mathrm{a} 5$ in the regulation of macrophage responses
\end{abstract}

Correspondence to: Professor Jianying Zhou, Department of Respiratory Medicine, The First Affiliated Hospital, Zhejiang University, 79 Qingchun Road, Hangzhou, Zhejiang 310003, P.R. China E-mail: zjyhz@zju.edu.cn

Dr Ran Chen, Zhejiang JFK Biological Technology Co., Ltd., 688 Binan Road, Building B Floor 2, Binjiang, Hangzhou, Zhejiang 310052, P.R. China

E-mail: rchen@fudan.edu.cn

${ }^{*}$ Contributed equally

Key words: Col4a5, macrophage, Mycobacterium tuberculosis, phagosomal acidification, vacuolar-type $\mathrm{H}^{+}$-ATPase to mycobacterial infection and identify Col4a5 as a potential target for the host-directed anti-mycobacterial therapy.

\section{Introduction}

Tuberculosis, caused by Mycobacterium tuberculosis (MTB), has affected one-third of the world's population; in 9 million individuals, this has developed into active infection and 1.5 individuals succumbed to the disease in 2013 according to WHO reports $(1,2)$. Notwithstanding advances in the diagnosis and treatment of tuberculosis, this disease remains a major cause of human morbidity and mortality due to the limitations of currently available therapies and vaccines, and the emergence and spread of multi-drug-resistant strains $(2,3)$. MTB has evolved a diverse number of strategies to evade host defense mechanisms, particularly in macrophages, by potentiating bacterial invasion and survival, and subverting host celldefending signaling pathways $(4,5)$. The successful survival strategies within macrophages employed by MTB include the reduction of the acidification and maturation of phagosomes that engulf the mycobacteria (6), preventing phagosomelysosome fusion (7), blocking autophagy-mediated killing (1), inducing inflammatory responses (8) and promoting cholesterol accumulation (9). Among the specific mechanisms targeted by MTB are signaling pathways mediated by mitogen-activated protein kinases (10), phosphatidylinositol 3-kinase (11), calcium $\left(\mathrm{Ca}^{2+}\right)(12)$ and cytokines, such as interleukin-1 and type I interferon (IFN) $(13,14)$.

Pathogens can exploit host genes to enter, reproduce in or exit from host cells or to circumvent host defenses $(1,15)$. Therefore, adjunctive host-directed therapies have the potential to augment cellular immune responses, to modify inflammatory processes and to enhance tuberculosis chemotherapy (16). Decreased interleukin-1 responses and exaggerated type I interferon production in MTB-infected mice have been shown to be associated with an eicosanoid imbalance, leading to an increased mortality of the mice (13). A broad range of hostdirected candidate agents, such as autophagy inducers, protein kinase inhibitors and antimicrobial peptide inducers, have been developed for clinical evaluation; however, none of these therapeutic agents have been approved for clinical use yet (16). The analysis of host genetic variations, which can alter suscepti- 
bility, may provide novel targets for host-directed therapies (15). These genetic variations can be identified by genome-wide gene inactivation strategies, including the use of siRNA/shRNA, miRNA and antisense/sense RNA libraries (17-19).

In the present study, we established an MTB-infection-based RAW264.7 macrophage screening model. First, we non-selectively inactivated genes using lentivirus-based RNA screening libraries, and isolated macrophage cell clones showing increased survival following MTB infection. DNA sequencing and bioinformatics analysis revealed several candidate host genes and an unsuspected role of collagen $\alpha-5$ (IV) chain (Col4a5), a type IV collagen subunit present on the cell surface, in mycobacterial infection. In addition, the analysis of the molecular mechanisms underlying the role of Col4a5 in MTB infection revealed the ability of this collagen to alter phagosomal acidification mediated by vacuolar-type $\mathrm{H}^{+}$-ATPase (V-ATPase) in murine macrophages. Our findings indicate that host-directed therapy targeting Col4a5 may represent an alternative therapeutic approach for combating MTB infection.

\section{Materials and methods}

$M T B$ and mycobacterium bovis Calmette-Guérin (BCG) cultures. The MTB strain, H37Rv, and the BCG strain, Pasteur, were grown to early log phase in Middlebrook $7 \mathrm{H} 9$ broth with BBL Middlebrook OADC (oleic acid, albumin, dextrose and catalase) enrichment medium (BD Pharminigen, San Diego, CA, USA) and 0.1\% (vol/vol) Tween-80 (Sigma-Aldrich, St. Louis, MO, USA). Predominantly single-cell suspensions were collected by centrifuging twice at $125 \mathrm{x} \mathrm{g}$ for $5 \mathrm{~min}$, re-suspension in phosphate-bovine serum (PBS) with $0.1 \%$ Tween-80, and re-centrifugation at $125 \mathrm{x}$ g for $10 \mathrm{~min}$, and used for the infection of macrophages.

Screening of macrophage host genes affecting MTB infection. Lentiviruses were produced by transiently transfecting 293 T cells [American Type Culture Collection (ATCC), Manassas, VA, USA] using a Random Homozygous Knock Out (RHKO) or an EST library DNA along with DNAs for the G418 (both from Sigma-Aldrich) resistance gene, packaging and VSVG envelope constructs, as demonstrated by us and others previously $(18,20,21)$. RAW264.7 macrophages (ATCC) were infected with purified lentiviruses and selected with $0.7 \mathrm{mg} /$ ml G418 (Invitrogen, Carlsbad, CA, USA). Approximately $1 \times 10^{7}$ G418-resistant clones of RAW264.7 macrophages were cultured at approximately $80 \%$ confluency, and exposed to MTB H37Rv at multiplicities of infection (MOI) of 2-3, 10-17 or 52-77 for 3 days, until the cells appeared dead under microscopic inspection. MTB was then killed with streptomycin $(20 \mu \mathrm{g} / \mathrm{ml})$, isoniazid $(5 \mu \mathrm{g} / \mathrm{ml})$ and rifampicin $(5 \mu \mathrm{g} / \mathrm{ml})$ (Sigma-Aldrich). Fresh DMEM (Invitrogen) plus $10 \%$ fetal bovine serum (FBS) (Atlanta Biologicals, Flowery Branch, GA, USA) were added to support the re-growth of surviving cells to approximately $80 \%$ confluency and used for re-infection. Following 3 cycles of infection and re-growth, cell colonies appeared. Single clones of cells were obtained by limited dilution and expansion in 96-well plates. Single clones stably transfected with antisense constructs were confirmed by genomic PCR with primers ESTF and ESTR, and analyzed for inserted sequences by DNA sequencing as described by us and others previously $(20,21)$.
In order to verify that the phenotypes of the isolated clones are due to the inactivation of the genes targeted by the RHKO or EST inserts, RNAi (shRNA-incorporated) and the vector-only control RAW264.7 macrophages with target genespecific knockdowns were established. Briefly, the RAW264.7 macrophages were seeded at approximately $5 \%$ confluency in fresh DMEM plus 10\% FBS, incubated for $24 \mathrm{~h}$, suspended and infected with filtered lentivirus in $5 \mathrm{mg} / \mathrm{ml}$ polybrene at $37^{\circ} \mathrm{C}$ for $6-18 \mathrm{~h}$ at a MOI retrospectively calculated as approximately 0.1 , followed by selection in puromycin (Invitrogen) at $2.5 \mu \mathrm{g} / \mathrm{ml}$ for $72 \mathrm{~h}$. A total of $1 \times 10^{7}$ puromycin-resistant clones was recovered after 10 days and the phenotype of target gene knockdown was confirmed using reverse transcriptoin-quantitative polymerase chain reaction (RT-qPCR) and/or western blot analysis.

$R T-q P C R$. RNA samples were prepared using Qiagen RNA extraction kits (Quiagen, Hilden, Germany). Primers for Col4a5 were AACGGGGGTTTCCAGGTTTAG (forward) and TTGGTTCCATTGCATCCAGG (reverse). Primers for Wrn (Werner syndrome ATP-dependent helicase) were GCCTGT TTACTTGGATCTGCACAG (forward) and TCATCC ACAGCAATGAGAGTGATG (reverse). Primers for glucuronidase beta (GUSB) were CCTGCGGTTGTGATGTGGTC (forward) and CCTCCAAATGCCCATAGTCATG (reverse). RNA was transcribed into cDNA with oligo-d(T) using MulLV reverse transcriptase (Applied Biosystems, Carlsbad, CA, USA). RT-qPCR reactions were performed using Bio-Rad SYBR kits and Col4a5 mRNA levels were normalized to GUSB mRNA levels.

Preparation of murine bone marrow-derived macrophages (BMMs). Young adult male C57BL/6 mice were purchased from the Zhejiang University animal facility. All animal experimental protocols used in this study were approved by Zhejiang University's Animal Care and Use Committee. BMMs were isolated and cultured by the following procedures: L929 cells (ATCC) were cultured with an initial 50\% confluency in RPMI-1640 medium (Invitrogen) plus 10\% FBS for 5 days, and the conditioned media were collected, filtered with a $0.22 \mu \mathrm{m}$ filter and stored as L-cell conditioned medium at $-20^{\circ} \mathrm{C}$. Bone Marrow Growth Medium containing RPMI1640 (Invitrogen) plus 20\% FBS, 30\% L-cell conditioned medium and $1 \%$ penicillin/streptomycin ( $\mathrm{P} / \mathrm{S})$ (Invitrogen), was prepared, aliquoted and stored at $-20^{\circ} \mathrm{C}$. The mice were sacrificed by cervical dislocation, and the pelvic and femoral bones were separated, immersed in $75 \%$ alcohol for $5 \mathrm{~min}$ followed by immersion in Dulbecco's phosphate-buffered saline (Invitrogen) for $5 \mathrm{~min}$, and left in RPMI-1640 (Invitrogen) plus $1 \% \mathrm{P} / \mathrm{S}$ (Invitrogen). The ends of the bones were cut and a $27 \mathrm{~g}$ needle/1 $\mathrm{ml}$ syringe filled with bone marrow growth medium was injected to expel the bone marrows from both ends of the bones and directed into a $15 \mathrm{ml}$ cell culture dish. The bone marrow cells were cultured and the medium was changed every 3 days. Some of the cells became substrate attached in culture, whereas most grew in suspension. The cells in suspension were spun-down and re-cultured in new dishes. After 10 days, almost all cells became attached and the BMMs were ready for further analysis. RNAi-treated BMMs were obtained by transient siRNA-transfection as previously reported by us (18). 
MTT assay. The mycobacteria were harvested, the OD600 of the mycobacterial culture was measured, and the bacterial number was calculated as $0.001 \mathrm{OD} 600=1 \times 10^{5} / \mathrm{ml}$, as previously described (22). The bacteria were mixed vigorously using a vortex, and diluted in tissue-culture medium to obtain the desired density to infect the macrophages at an MOI of 3 or 9 . Bacterial colony forming units (CFU) from infected cultures were determined at the beginning and end of each experiment in 7H10 medium supplemented with OADC. RAW264.7 monolayers infected with the MTB strains were incubated at $37^{\circ} \mathrm{C}$ and $5 \% \mathrm{CO}_{2}$ for up to $72 \mathrm{~h}$. After supernatant removal, the wells were incubated with 3-(4,5-dimethylthiazol-2-yl)-2,5-diphenyltetrazolium bromide [MTT (Sigma-Aldrich), $5 \mathrm{mg} / \mathrm{ml}$ in PBS] with RPMI-1640 medium for further $2 \mathrm{~h}$. The resulting formation of formazan crystals in the cells was documented, and the cultures were then dissolved in $100 \mu \mathrm{l}$ dimethyl sulfoxide. The absorbance of the purple formazan product was measured at $490 \mathrm{~nm}$ with a reference wavelength of $620 \mathrm{~nm}$ using a microplate reader (Tecan, Shanghai, China). All experiments were performed in quadruplicate and the relative cell viability (\%) was expressed as percentage relative to the untreated control cells.

CFU determination for intracellular mycobacteria. The mycobacterial cultures were centrifuged at $125 \mathrm{x} \mathrm{g}$ for $10 \mathrm{~min}$ at room temperature, pellets were re-suspended in RPMI-1640, and then applied to macrophage cultures at an MOI of 3 or 9 for 6,30 or $72 \mathrm{~h}$ at $37^{\circ} \mathrm{C}$ in a $0.5 \% \mathrm{CO}_{2}$ incubator. The monolayers were washed with warm PBS to remove extracellular bacteria and re-cultured in RPMI-1640 (nutrient-enriched, 'GM' condition) or PBS (starvation, 'ST' condition) containing $20 \mu \mathrm{g}$ / $\mathrm{ml}$ gentamycin to inhibit the growth of extracellular/released bacteria in infected wells. At various time points, the medium was aspirated from infected macrophage wells and $100 \mu \mathrm{l}$ of sterile lysis buffer $\left(0.05 \%\right.$ SDS in $\left.\mathrm{ddH}_{2} \mathrm{O}\right)$ were added to each well. The plates were incubated at room temperature for $5 \mathrm{~min}$, serial dilutions were carried out, and $0.1 \mathrm{ml}$ volumes were plated for 3 dilutions in duplicate on 7H11 agar plates and further incubated at $37^{\circ} \mathrm{C}$ for 21 days. CFUs of intracellular mycobacteria were then counted.

Western blot analysis. The RAW264.7 cells were seeded in plates and infected with or without BCG at the MOIs described above. At the indicated time points, the macrophages were lysed and boiled in reducing sodium dodecyl sulfate-polyacrylamide gel electrophoresis (SDS-PAGE) sample buffer, subjected to 4-15\% gradient gel electrophoresis (Bio-Rad, Hercules, CA, USA) and transferred onto a $0.2 \mu \mathrm{m}$ nitrocellulose membrane (GE Healthcare, Piscataway, NJ, USA) as previously described (23). The blots were probed with antiV-ATPase subunit B antibody (Cat. no. SC-271832; Santa Cruz Biotechnology, Inc., Santa Cruz, CA, USA), anti-Col4a5 antibody (Cat. no. ABIN375285; Antibodies-online, Aachen, Germany), and anti-tubulin (Cat. no. T3526), and anti-V-ATPase subunit E (Cat. no. HPA016938) antibodies (Sigma-Aldrich). The detection of immunoreactivity was performed using the ECL (New England Bioscience, Ipswich, MA, USA) or Odyssey detection system (LI-COR Biosciences, Lincoln, NE, USA).

Immunofluorescence. The macrophages were fixed with $4 \%$ paraformaldehyde in PBS for $2 \mathrm{~h}$ and permeabilized with
$0.2 \%$ saponin in PBS containing $10 \%$ donkey serum (SigmaAldrich) for $30 \mathrm{~min}$ at room temperature. The coverslips were incubated for $30 \mathrm{~min}$ at room temperature with anti-Col4a5 antibody (dilution, 1:50; Cat. no. ABIN375285), rinsed twice with PBS containing $0.2 \%$ saponin, and stained for $1 \mathrm{~h}$ at room temperature with secondary antibody (dilution, 1:500) conjugated with TexRed (Molecular Probes, Carlsbad, CA, USA). The coverslips were washed twice with PBS containing $0.2 \%$ saponin and once in $\mathrm{ddH}_{2} \mathrm{O}$, and mounted with DAPI on slides. Images were acquired with an inverted Laser scanning confocal microscope (LSM 510; Leica Microsystems, Wetzlar, Germany).

Measurement of the endosomal/lysosomal pH in macrophages. Dextran-Oregon Green 488 is an endosomal and lysosomal marker traveling in the cell via the endosomal/ lysosomal routes. The ratio of the fluorescence at excitation 490 to $440 \mathrm{~nm}$ correlates with endosomal/lysosomal $\mathrm{pH}$ values. Using standard curve-fitting, $\mathrm{pH}$ values can be calculated from these fluorescence ratios (24). For fluorescence determinations, the RAW264.7 cells were plated to $60-70 \%$ confluency on fluorescence-proof microplates (Corning, New York, NY, USA). To measure the endosomal/lysosomal $\mathrm{pH}$ values, the cells were incubated with $0.5 \mu \mathrm{g} / \mathrm{ml}$ Dextran-Oregon Green 488 (Molecular Probes) for $30 \mathrm{~min}$ to $5 \mathrm{~h}$ at $37^{\circ} \mathrm{C}$. The fluorescence of Dextran-Oregon Green 488 was measured with the excitation wavelength set at 440 and $490 \mathrm{~nm}$, respectively, and the fluorescence emission set at $520 \mathrm{~nm}$ using a microplate reader (Tecan). The fluorescence ratio of 490/440 was converted to $\mathrm{pH}$ values by standard curve-fitting as reported previously (25). Briefly, the cells in the plate were incubated with Dextran-Oregon Green 488 for $1 \mathrm{~h}$ and then incubated in isotonic $\mathrm{K}^{+}$-rich medium buffered to predetermined $\mathrm{pH}$ values (between $\mathrm{pH} 7.0$ and 3.5). Alternatively, the membranes were permeabilized gently using a solution of $0.1 \%$ Triton X-100 in PBS for $2 \mathrm{~min}$, given direct access to the Dextran-Oregon Green 488 particles. Calibration curves were obtained by plotting the extracellular $\mathrm{pH}$, which is assumed to be identical to the internal $\mathrm{pH}$, against the corresponding fluorescence ratio.

Measurement of phagosomal pH in macrophages. Phagosomal $\mathrm{pH}$ values were measured as previously described (26). Briefly, BCG was labeled by incubation in N-Hydroxysuccinimidyl ester 5- (and -6)-carboxyfluorescein (NHS-CF, $0.1 \mathrm{mg} / \mathrm{ml}$ ) (Sigma-Aldrich) and FITC (1 mg/ml) (Sigma-Aldrich) on ice for $30 \mathrm{~min}$. Unbound dye was removed by repeated washing. The bacteria were heated at $100^{\circ} \mathrm{C}$ for $10 \mathrm{~min}$; the heat-killed bacteria were used for comparison. The RAW264.7 cells were incubated with FITC-BCG on fluorescence-proof microplates (Corning) for 1-5 h. Measurements of phagosomal $\mathrm{pH}$ were obtained through the fluorescence ratio reading method as described for endosomal/lysosomal $\mathrm{pH}$ measurements. The calibration of the fluorescence ratio versus the $\mathrm{pH}$ value was performed for each experiment by equilibrating the cells in isotonic $\mathrm{K}^{+}$-rich medium buffered to varying $\mathrm{pH}$ values (between $\mathrm{pH} 4.0$ and 8.0) in the presence of the $\mathrm{K}^{+} / \mathrm{H}^{+}$ionophores nigericin (5 $\mu \mathrm{M}$; Sigma-Aldrich) and monensin $(2 \mu \mathrm{M}$; Sigma-Aldrich). Calibration curves were obtained by plotting the extracellular $\mathrm{pH}$, which is assumed to be identical to the internal $\mathrm{pH}$, against the corresponding fluorescence ratio. 
Table I. Putative host target genes involved in the regulation of tuberculosis infection.

\begin{tabular}{|c|c|c|c|}
\hline Gene & Library/direction & MOI used & Annotation \\
\hline Col4a5 & RHKO/antisense & $2-3$ & Collagen IV subunit \\
\hline eIF4e & RHKO/antisense & $10-17$ & Eukaryotic translation initiation factor $4 \mathrm{E}$ \\
\hline Mllt3 & RHKO/sense & $2-3,10-17$ & $\begin{array}{l}\text { Myeloid/lymphoid or mixed-lineage leukemia } \\
\text { (trithorax homolog, Drosophila) }\end{array}$ \\
\hline Pgm211 & RHKO/sense & $2-3,10-17$ & Phosphoglucomutase 2 -like 1 \\
\hline Wrn & RHKO/antisense & $2-3,10-17$ & Werner syndrome, RecQ helicase-like \\
\hline Zfp52 & RHKO/antisense & $2-3$ & Mouse zinc finger protein 52 \\
\hline NM_182745 & RHKO/sense & $2-3,10-17$ & Hypothetical protein, function unknown \\
\hline PLBD1 & EST/antisense & $10-17$ & Phospholipase B domain containing 1 \\
\hline F7 & EST/antisense & $10-17$ & Coagulation factor VII \\
\hline EXTL2 & EST/antisense & $52-77$ & Exostoses (multiple)-like 2 \\
\hline LMTK2 & EST/antisense & $52-77$ & Lemur tyrosine kinase 2 \\
\hline DPEP1 & EST/antisense & $52-77$ & Dipeptidase 1 (renal) \\
\hline Sp100 & EST/antisense & $52-77$ & SP100 nuclear antigen \\
\hline PKR & EST/antisense & $52-77$ & EIF2AK2, eukaryotic translation initiation factor $2-\alpha$ kinase 2 \\
\hline CST8 & $\mathrm{EST} /$ sense & $52-77$ & Cystatin 8 (cystatin-related epididymal spermatogenic) \\
\hline AL137616 & $\mathrm{EST} /$ sense & $52-77$ & Unknown \\
\hline ACADVL & $\mathrm{EST} /$ sense & $10-17$ & Acyl-CoA dehydrogenase, very long chain \\
\hline PDE6B & $\mathrm{EST} /$ sense & $10-17$ & Phosphodiesterase 6B, cGMP, rod receptor, beta polypeptide \\
\hline CNKSR2 & $\mathrm{EST} /$ sense & $52-77$ & Connector enhancer of kinase suppressor of Ras \\
\hline ARMC9 & $\mathrm{EST} /$ sense & $10-17$ & Armadillo repeat containing 9 \\
\hline Hdac9 & $\mathrm{EST} /$ sense & $10-17$ & Histone deacetylase 9 \\
\hline Runx1 & $\mathrm{EST} /$ sense & $52-77$ & Runt-related transcription factor 1 \\
\hline Spink1 & $\mathrm{EST} /$ sense & $52-77$ & Serine peptidase inhibitor, Kazal type 1 \\
\hline Rnf135 & $\mathrm{EST} /$ sense & $10-17$ & Ring finger protein 135 \\
\hline TRAIP & $\mathrm{EST} /$ sense & $10-17$ & TRAF interacting protein \\
\hline Pak1 & $\mathrm{EST} /$ sense & $10-17$ & p21 protein $(\mathrm{Cdc} 42 / \mathrm{Rac})$-activated kinase 1 \\
\hline
\end{tabular}

MOI, multiplicity of infection; Col4a5, collagen $\alpha-5$ (IV) chain.

Measurement of microsomal V-ATPase activity in macrophages. ATPase-mediated ATP hydrolysis is coupled enzymatically to the oxidation of $\mathrm{NADH}$, which can be followed by an absorbance decrease at $340 \mathrm{~nm}$; therefore, V-ATPase activity was measured using a modification of the protocol previously described (26). Briefly, microsomal fractions were obtained by ultracentrifugation of a macrophage homogenate using a sucrose gradient, and $10 \mu \mathrm{g}$ of microsomal protein were added to a master mix containing $37.5 \mathrm{mM}$ MOPS, $4 \mathrm{mM} \mathrm{MgSO}_{4}, 50 \mathrm{mM} \mathrm{KCl}, 3 \mathrm{mM}$ orthovanadate, $1 \mathrm{mM}$ phosphoenolpyruvate, $0.3 \mathrm{mM} \mathrm{NADH}, 10 \mathrm{mg} / \mathrm{ml}$ pyruvate kinase and $10 \mathrm{mg} / \mathrm{ml}$ lactate dehydrogenase (Sigma-Aldrich). Reactions with and without the V-ATPase-specific inhibitor bafilomycin A1 (BA1; 100 nM; Sigma-Aldrich) were started by the addition of ATP to a final concentration of $2 \mathrm{mM}$ in a microtiter plate, and absorbance at $340 \mathrm{~nm}$ was monitored for $20 \mathrm{~min}$ in a Safire plate reader (Tecan). All measurements were performed in triplicate, and V-ATPase activity was calculated as BA1-inhibited ATP hydrolysis.

Statistical analysis. All experiments were repeated 3 times independently and the data were presented as the means \pm SEM.
One-way analysis of variance followed by Dunnett's test were used to determine significant differences. Significant levels were set at $\mathrm{P}<0.05$. Statistical analyses were performed using SPSS statistical software (version 21.0; SPSS, Inc., Chicago, IL, USA).

\section{Results}

Knockdown of host target genes increases the viability of MTB-infected macrophages. RAW264.7 cell libraries in which cellular genes were randomly inactivated by lentivirus-based antisense RNA methods were constructed using the RHKO and EST DNA libraries, as previously reported by us $(18,21)$. These macrophage libraries were screened for clones that survived multiple cycles of infection by virulent MTB (H37Rv) at moderate to high MOI values. Individual surviving macrophage cell clones were expanded, and DNA sequencing identified 26 unique, inserted DNA sequences in a total of 81 macrophage clones (Table I). Among these clones, the Wrn mutant survived in the highest frequency from 3 cycles of the MTB infection and was the only mutant surviving after 4 cycles of MTB infection. A series of RT-qPCR assays confirmed the downregulation of the host target genes in the isolated macro- 


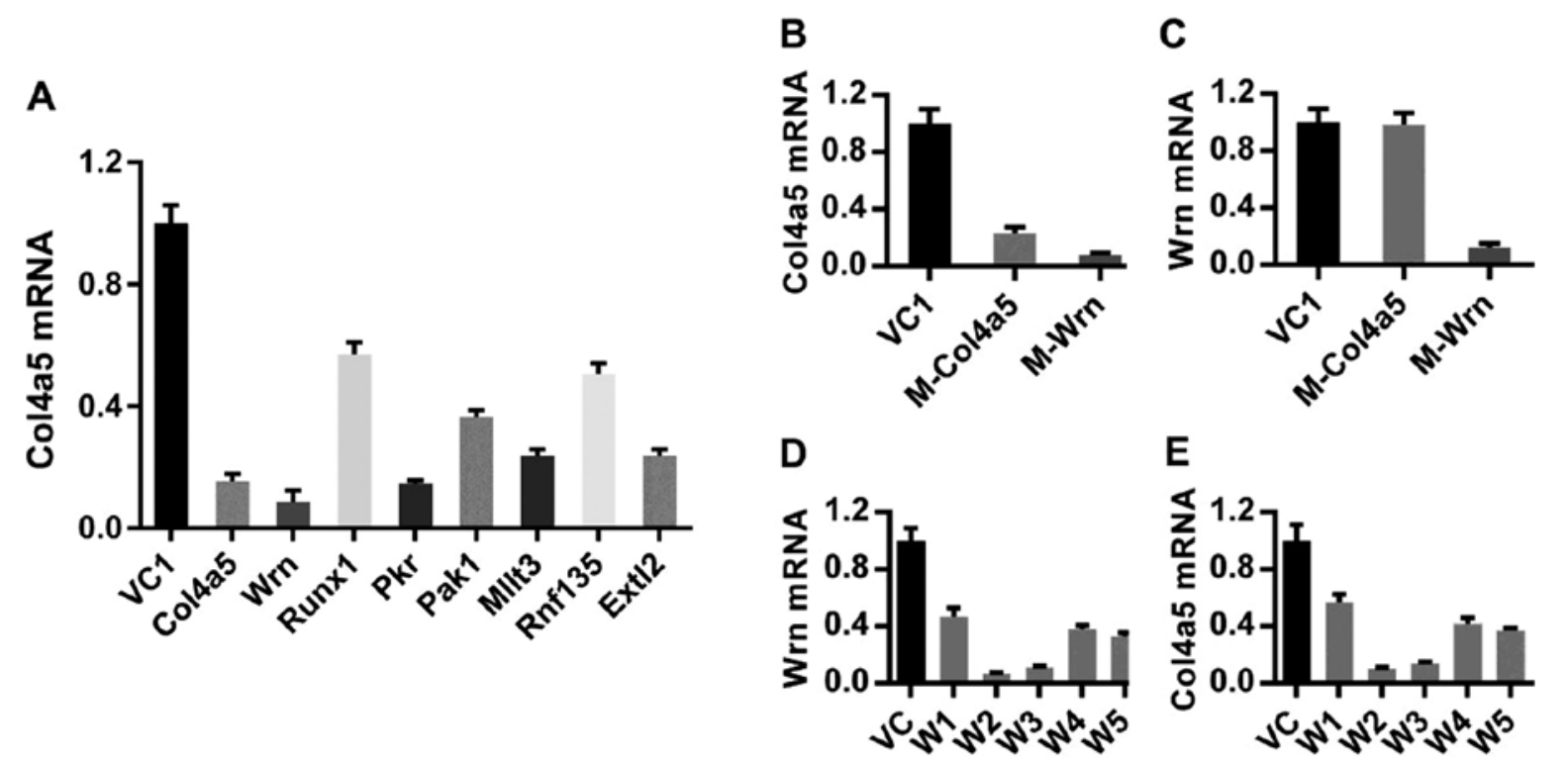

Figure 1. Identification and validation of host target genes affecting Mycobacterium tuberculosis (MTB) infection function as regulators of collagen $\alpha-5$ (IV) chain (Col4a5) expression. (A) Relative quantification of Col4a5 mRNA expression in vector control (VC1), Col4a5, Wrn, Runx1, Pkr, Pak1, Mllt3, Rnf135 and Extl2 mutant cells was determined by RT-qPCR. Quantification of relative (B) Col4a5 mRNA and (C) Wrn mRNA levels determined by RT-qPCR in VC1, Col4a5-mutant (M-Col4a5) and Wrn-mutant (M-Wrn) cell lines. Quantification of relative (D) Wrn mRNA or (E) Col4a5 mRNA levels determined by RT-qPCR in VC1 and 5 Wrn-shRNA-transfected cell lines (W1, W2, W3, W4 and W5).

phage clones and additionally revealed that the transcription of one of the 26 host target genes, Col4a5, was downregulated in clones containing antisense inserts in several of the host target genes, including Wrn, Runx1, Pkr, Pak1, Mllt3, Rnf135 and Extl2 mutants (Fig. 1A). These results led us to speculate that the inserts identified by our screening may affect a common pathway regulating Col4a5 expression. Consistent with this notion, Col4a5 was downregulated in Wrn mutant cells (Fig. 1B), while the expression of Wrn was not altered in Col4a 5 mutant cells (Fig. 1C). Moreover, the expression levels of the Runx1, Pkr, Pak1, Mllt3, Rnf135 and Extl2 genes were not significantly altered in the Col4a5 mutant cells (data not shown). Col4a5 was also downregulated in 5 Wrn-shRNA-incorporated lines and correlated with the decreased Wrn mRNA levels in those cell lines (Fig. 1D and E), confirming that Wrn is a positive regulator of Col4a5 expression at the transcriptional level. Additionally, genes, such as IFN $\gamma$, known to affect MTB infection may alter Col4a 5 expression or function at the transcriptional and/or posttranscriptional levels $(27,28)$. These findings led us to explore a possible role of Col4a5 as a target gene for the host-directed therapy of mycobacterial infection.

Col4a5 deficiency promotes macrophage survival following mycobacterial infection. In order to further confirm the effects of Col4a5 on mycobacterial infection, the knockdown of Col4a5 by shRNA was employed in the RAW264.7 macrophages. To mitigate possible off-target effects of the antisense RNA-based methods used to identify Col4a5, 3 separate Col4a5-shRNA-transfected macrophage lines, designated as C51, C52 and C53, were established, each of which contained shRNA species targeting a different locus of the Col4a5 mRNA. The results of RT-qPCR revealed that the Col4a5 mRNA levels in the C51, C52 and C53 cells were decreased to approximately $15-20 \%$ of the level observed for the vector-only control (VC) cells (Fig. 2A). Consistent with the reduction in mRNA expression, the results of western blot analysis confirmed decreased Col4a5 protein levels in the C51, C52 and C53 cells compared with the VC cells (Fig. 2B). Furthermore, western blot analysis andd immunofluorescence staining revealed decreased Col4a 5 protein staining in the cell membranes for all 3 cell lines (Fig. 2B and C). Furthermore, the growth rates of Col4a5-deficient cell lines were similar to those of the control cells without MTB infection, as indicated by the cell numbers on days 1 and 3 (Fig. 3A). In addition, the deficiency of Col4a5 in the macrophages was associated with an enhanced survival following infection by MTB at either an MOI 3 or 9 on day 3. MTT assays consistently revealed that after 3 days of MTB infection, only approximately $10 \%$ of the vector-control cells survived, while $>40 \%$ of the macrophages in which Col4a5 was knocked down survived under the same culture conditions (Fig. 3B). Taken together, these results suggested that Col4a5 deficiency enhanced macrophage viability following mycobacterial infection.

Col4a5 deficiency suppresses the intracellular mycobacterial viability in macrophages. To further investigate the effects of Col4a5 deficiency on the viability of intracellular MTB, the intracellular mycobacterial survival rate was estimated by $\mathrm{CFU}$ counting. Macrophages were infected with MTB at an MOI of 3 in either nutrient-enriched (GM) or starvation (ST) conditions. The knockdown of Col4a5 led to an approximately $20-80 \%$ decrease in MTB numbers recovered from the host cells incubated for 6, 30 or $72 \mathrm{~h}$ after infection (Fig. 4A-C). When the MOI of MTB was increased to 9 , the knockdown of Col4a5 still led to an approximately 10-80\% decrease in MTB numbers recovered from the host cells for 6,30 or $72 \mathrm{~h}$ post-infection under nutrient-enriched or starvation conditions (Fig. 4D-F). Moreover, when siRNA transfection was employed to knockdown Col4a5 expression in BMMs, which was confirmed by both RT-qPCR and western blot analysis (Fig. 5A and B), 


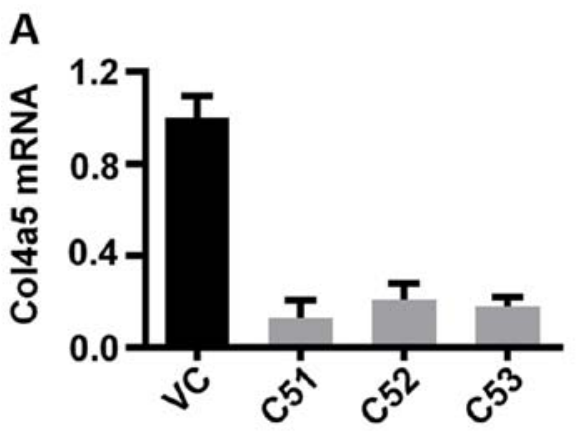

B

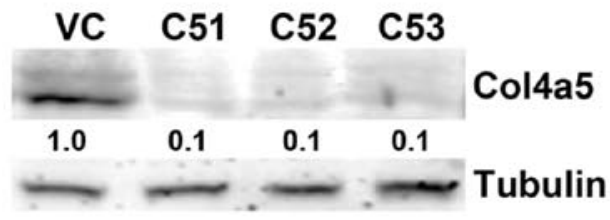

C

VC

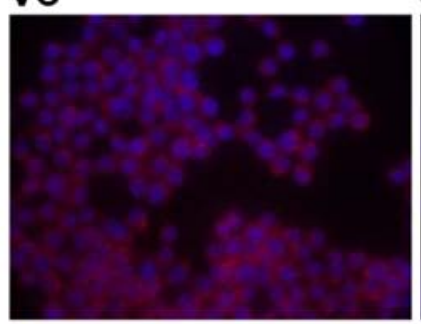

C51

C52

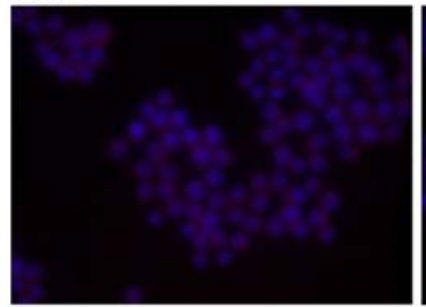

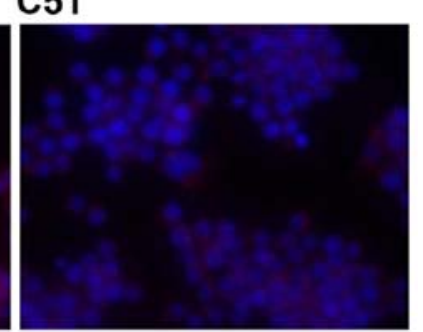

C53

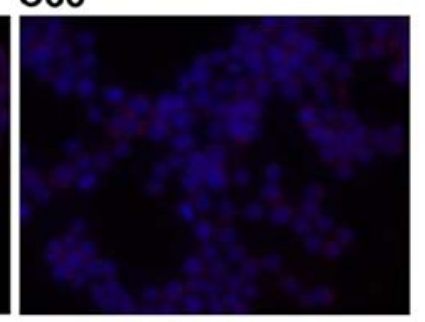

Figure 2. Confirmation of collagen $\alpha-5$ (IV) chain (Col4a5) knockdown in RAW264.7 macrophages. (A) Quantification of relative Col4a5 mRNA levels in vector control (VC), and 3 Col4a5-shRNA-transfected macrophage cell lines (C51, C52 and C53) determined by RT-q PCR. (B) Western blot analysis showing Col4a5 protein levels isolated from VC, C51, C52 and C53 cell lines. Tubulin served as an internal control. (C) Immunofluorescence staining revealed the expression of Col4a5 protein localized at cell membranes in VC, C51, C52 and C53 macrophage cell lines (red signal is Col4a5; blue signal represents DAPI-staining of nuclei).
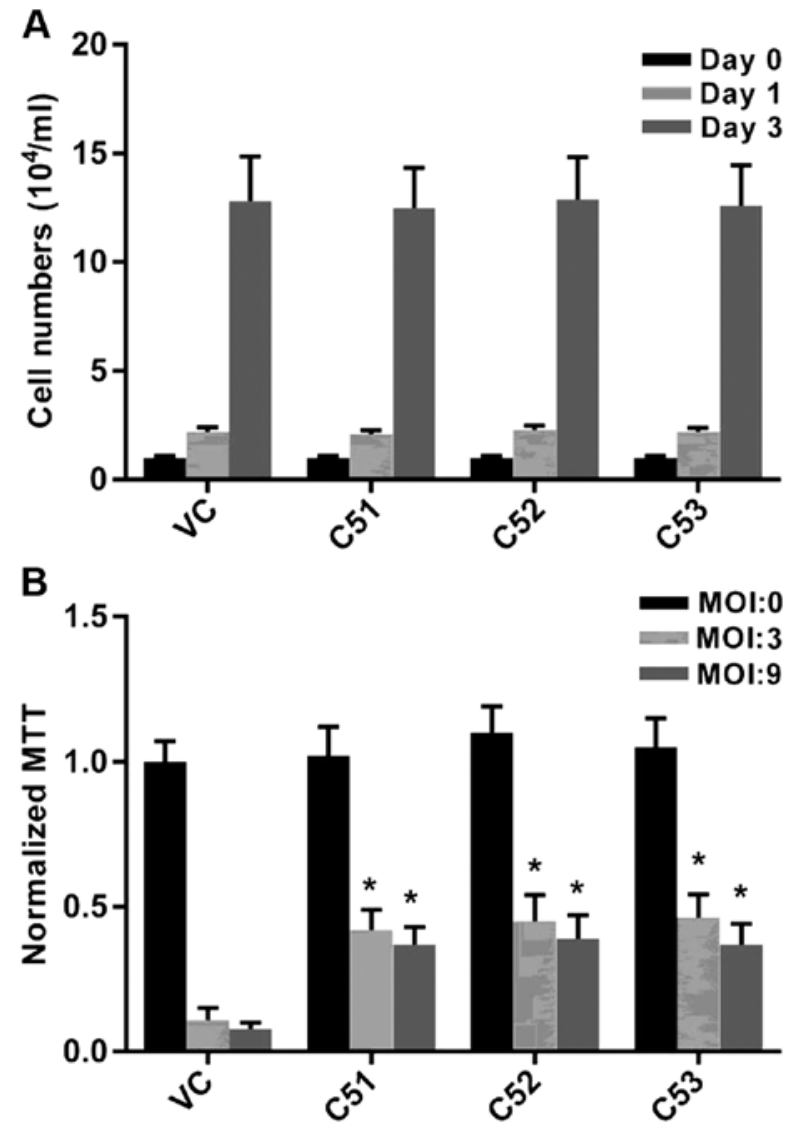

Figure 3. Effects of collagen $\alpha-5$ (IV) chain (Col4a5) knockdown on growth and viability in RAW264.7 macrophages. (A) Control (VC) and Col4a5-knockdown (C51, C52 and C53) macrophage cell lines were cultured and the cells numbers were counted on the seeding day (day 0), after overnight culture (day 1), and after 3 days in culture (day 3). (B) VC and cells in which Col4a5 had been knocked down (C51, C52 and C53) were not infected (MOI 0) or infected with Mycobacterium tuberculosis (MTB) at an MOI of 3 or 9. Four hours after infection, all cell lines were washed, cultured for 3 days, and examined by MTT assay. ${ }^{*} \mathrm{P}<0.05$ compared with VC.
Col4a5 knockdown led to an approximately 40-80\% decrease in MTB numbers recovered from the host cells $6 \mathrm{~h}$ post-infection under nutrient-enriched or starvation conditions (Fig. 5C). Collectively, these results suggested that Col4a5 knockdown led to the suppression of intracellular mycobacterial viability in vitro under both nutrient-enriched or starvation conditions.

Knockdown of Col4a5 enhances the acidification of both culture media and intracellular vesicles. Macrophages represent the first line of defense against MTB invasion by various mechanisms, a major one of which is the acidification of phagosomes and lysosomes $(29,30)$. During our studies, we observed that Col4a5 knockdown in macrophages was associated with a yellowing of the culture media, suggesting a possible decrease in $\mathrm{pH}$ of the culture media $\left(\mathrm{pH}_{\mathrm{m}}\right)$. The direct measurement of $\mathrm{pH}_{\mathrm{m}}$ values confirmed a 0.4-0.5 $\mathrm{pH}$ unit decrease (data not shown) for media in which the C51, C52 or C53 cells were grown for 3 days compared to the controls with the same growth rate as Col4a5-deficient cells (Fig. 3A). To further investigate the alterations in intravesicular $\mathrm{pH}$ ( $\mathrm{pH}_{\mathrm{i}}$, i.e., in endosomes, lysosomes and phagosomes) in macrophages, Dextran-Oregon Green 488, which is known to enter cells via endocytosis within $1 \mathrm{~h}$ and enter the lysosome within approximately $5 \mathrm{~h}$, was used to determine whether the deficiency of Col4a5 is associated with changes in $\mathrm{pH}_{\mathrm{i}}$. The endosomal $\mathrm{pH}$ was approximately 0.5 units lower and the lysosomal $\mathrm{pH}$ was approximately 0.9 units lower in the macrophages in which Col4a5 had been knocked down compared with the controls (Fig. 6A). FITC-labeled BCG were used to measure the phagosomal $\mathrm{pH}$, which was approximately 0.9 units lower in the cells in which Col4a 5 had been knocked down compared with the control macrophages (Fig. 6B). These results confirmed that Col4a5 knockdown enhances the the acidification of intracellular vesicles in murine macrophages. 
A

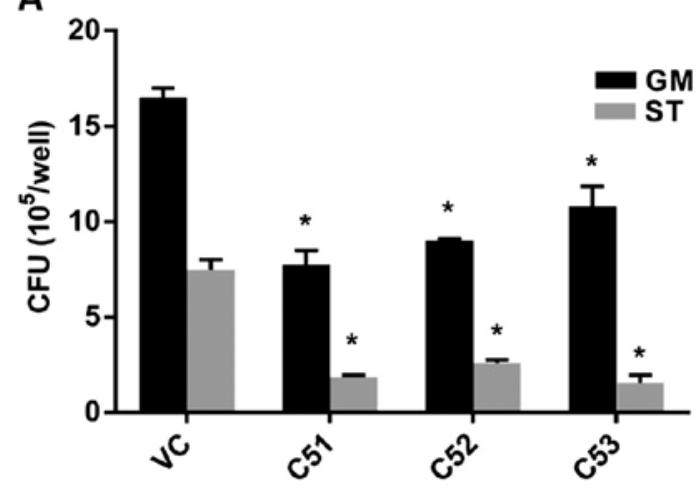

B

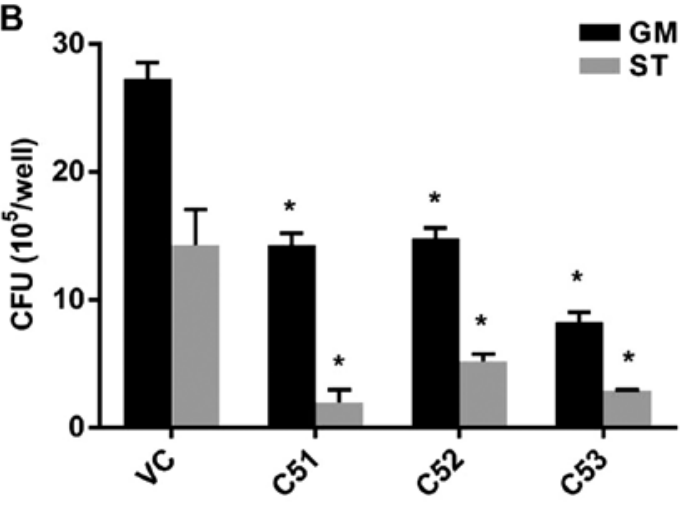

C

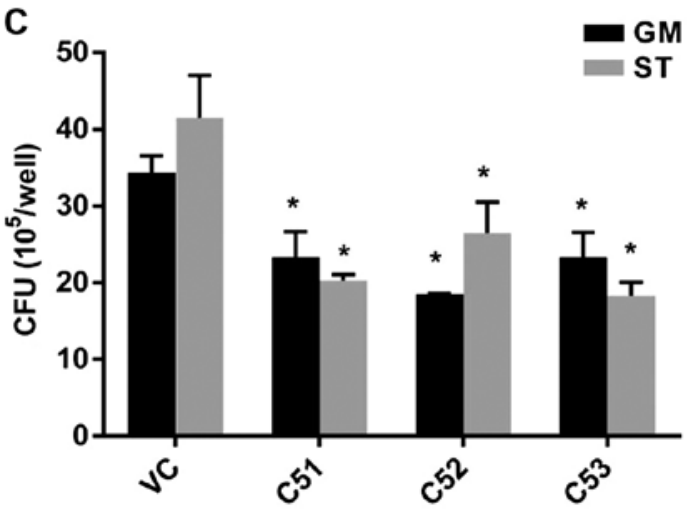

D
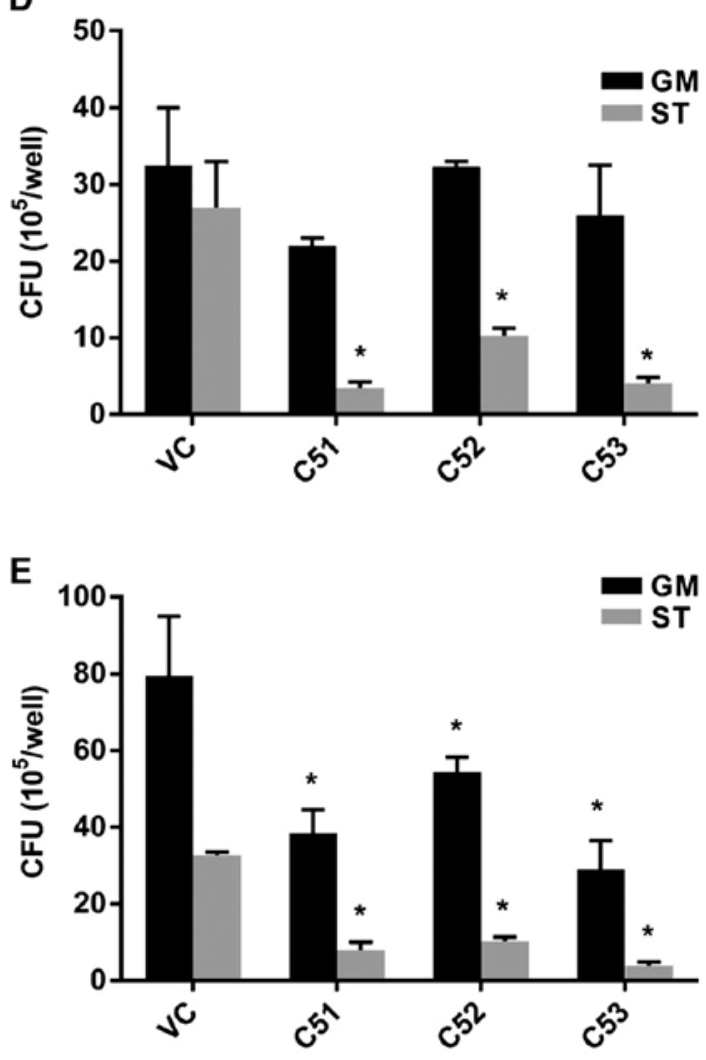

$\mathbf{F}$

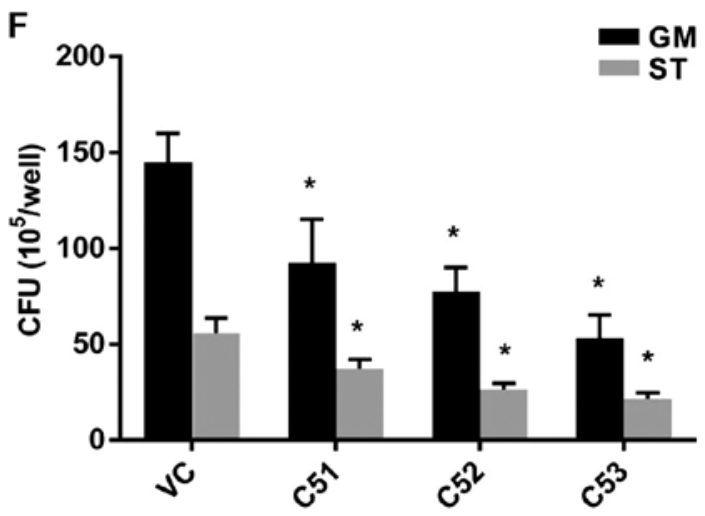

Figure 4. Effects of collagen $\alpha-5$ (IV) chain (Col4a5) knockdown on intracellular mycobacterial viability in RAW264.7 macrophages. (A-C) Control (VC) and cells in which Col4a5 had been knocked down (C51, C52 and C53) were infected with Mycobacterium tuberculosis (MTB) at an MOI of 3 and incubated for (A) $6 \mathrm{~h},(\mathrm{~B}) 30 \mathrm{~h}$ (B) and (C) $72 \mathrm{~h}$ under either nutrient-enriched (GM) or starvation conditions (ST). (D-F) VC and cells in which Col4a5 had been knocked down (C51, C52 and C53) were infected with MTB at an MOI of 9 and incubated for (D) $6 \mathrm{~h}$, (E) $30 \mathrm{~h}$ and (F) $72 \mathrm{~h}$ under either nutrient-enriched or starvation conditions. The numbers of MTB recovered from cell line lysates at different time points were measured as colony forming units (CFUs) "P<0.05 compared with VC.

Increased microsomal V-ATPase activity occurs post-translationally in cells in which Col4a5 is knocked down. MTB is known to protect itself from digestion by lysosomal enzymes by preventing the lowering of the $\mathrm{pH}$ necessary for the activity of lysosomal enzymes (31). Moreover, macrophages are known to actively secrete protons into the extracellular environment via the V-ATPase, which is crucial for maintaining intravesicular acidification (32). We hypothesized that the lower $\mathrm{pH}_{\mathrm{m}}$ and $\mathrm{pH}_{\mathrm{i}}$ of macrophages in which Col4a5 had been knocked down may be mediated via the actions of V-ATPase. Treatment of the cell cultures with BA1, a V-ATPase specific inhibitor, eliminated the $\mathrm{pH}_{\mathrm{m}}$ difference in the media (data not shown), confirming the possible role of V-ATPase for Col4a5-mediated acidification. Therefore, we measured the total ATPase activity by a quantitative assay and found that microsomal V-ATPase activity in the cells in which Col4a5 had been knocked down was approximately 5.5 -fold higher compared with that in the control cells (Fig. 7A). Moreover, in the presence of the V-ATPase specific inhibitor, BA1, the difference in BCG infection between the cells in which Col4a5 had been knocked down and the control cells was diminished (Fig. 7B). To further address whether the upregulation of V-ATPase activity was due to the expression of V-ATPase subunits, western blot analysis was performed detecting the 


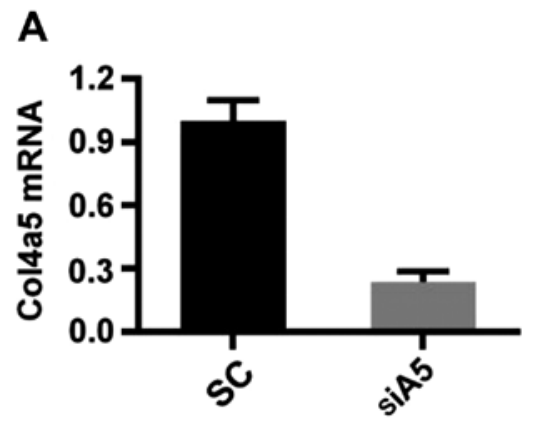

B

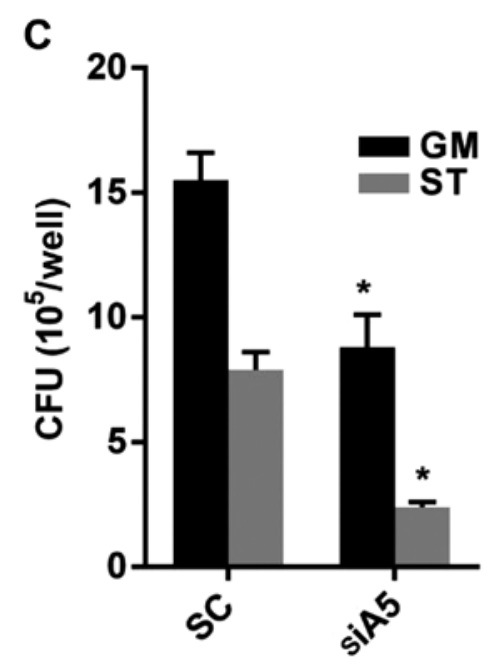

Figure 5. Effects of Col4a5 knockdown on intracellular mycobacterial viability in bone marrow-derived macrophages (BMMs). Col4a5 siRNA (siA5) or scrambled siRNA (SC) was transfected into BMMs. (A) Quantification of relative Col4a5 mRNA levels in SC- and siA5-transfected BMMs were determined by RT-qPCR. (B) Western blot analysis detected protein bands of Col4a5 isolated from SC- and siA5-transfected BMMs. Tubulin was used as an internal control. (C) SC- or siA5-transfected BMMs were infected with Mycobacterium bovis Calmette-Guérin (BCG) at an MOI of 3 and incubated for $6 \mathrm{~h}$ under either nutrient-enriched or starvation conditions and BCG levels recovered from cell lysates of BMMs were measured as colony forming units (CFUs). ${ }^{*} \mathrm{P}<0.05$ compared with SC.
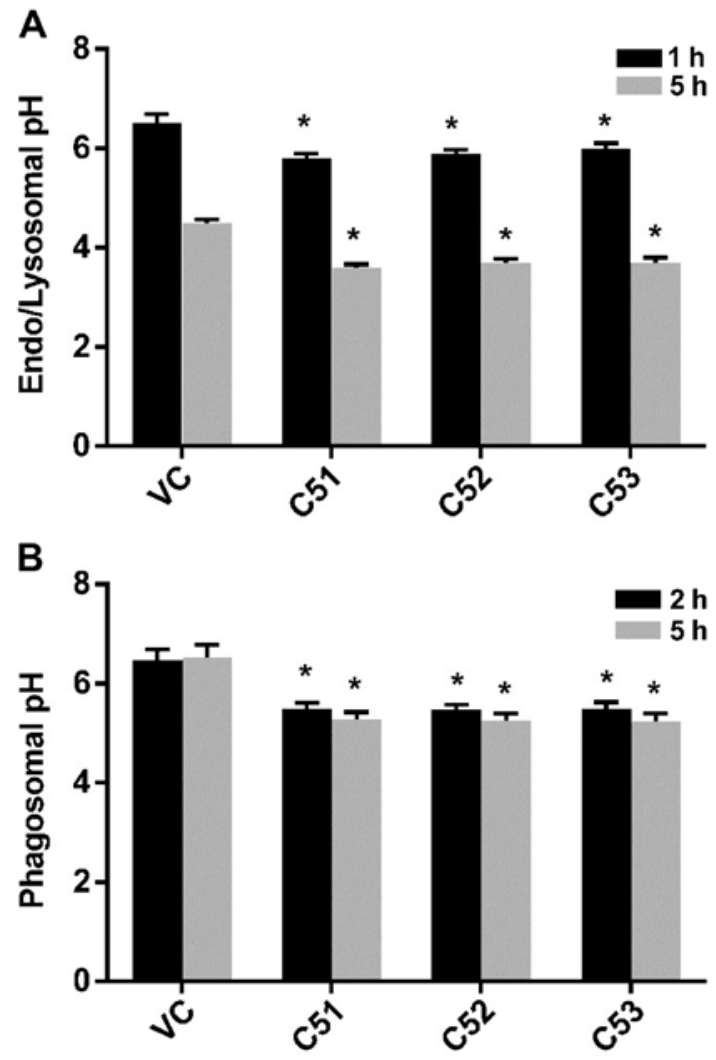

Figure 6. Effects of collagen $\alpha-5$ (IV) chain (Col4a5) knockdown on the acidification of intracellular vesicles in RAW264.7 macrophages. (A) DextranOregon Green 488 was added to the culture of control (VC) cells and cells in which and Col4a5 had been knockdown (C51, C52 and C53). After 1 and $5 \mathrm{~h}$, the fluorescence was measured at the excitation wavelength 490 and $440 \mathrm{~nm}$ and converted to $\mathrm{pH}$ values via standard curve fitting. (B) Mycobacterium bovis Calmette-Guérin (BCG)-FITC was added to the culture of VC, C51, C52 and C53 macrophage cell lines. After 2 and $5 \mathrm{~h}$, the fluorescence was measured at the excitation wavelength of 490 and $440 \mathrm{~nm}$ and converted to $\mathrm{pH}$ values via standard curve fitting. " $\mathrm{P}<0.05$ compared with VC.
V-ATPase subunits B and E in controls and in the macrophages in which Col4a5 had been knocked down without BCG infection (MOI 0) or with BCG infection (MOI 2 or 8). Col4a5 knockdown did not upregulate the protein expression of the ATPase subunits (Fig. 7C), suggesting that increased microsomal V-ATPase activity occurs post-translationally in cells in which Col4a5 is knocked down.

\section{Discussion}

In the present study, we screened for host genes which are exploited by MTB during invasion and survival in murine macrophages, and we identified Col4a5, a collagen IV subunit, as a potential host target gene. In addition, we found 9 host genes as positive regulators of Col4a5 gene expression. The knockdown of Col4a5 reduced mycobacterial viability and increased the survival of macrophages by promoting the acidification of intracellular vesicles by increasing V-ATPase activity.

Tuberculosis remains one of the major causes of mortality worldwide due to the lack of effective therapies and the emergence of multidrug-resistant strains (2). In addition to conventional chemotherapies, alternative host-directed therapies against interleukin-1 and type I IFN have been explored to improve treatment efficacies and to reduce the mortality rate associated with the disease $(13,33)$. Numerous host genes for MTB have been identified by RNAi screens against all known kinases and phosphatases in a mouse macrophage cell line (34) and against all genes in a human macrophage cell line (19); however, all these screens were based on reducing the intracellular mycobacterial load. In this study, we investigated host genes exploited by MTB during their invasion and survival in murine macrophages and identified Col4a5 as a potential host target gene. By contrast, the screening method we used to non-selectively inactivate genes in RAW264.7 cells 
A

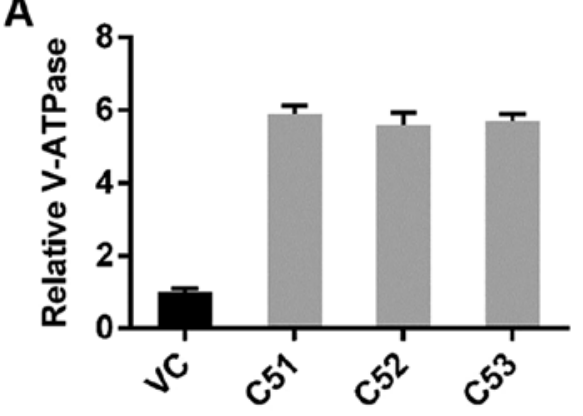

B

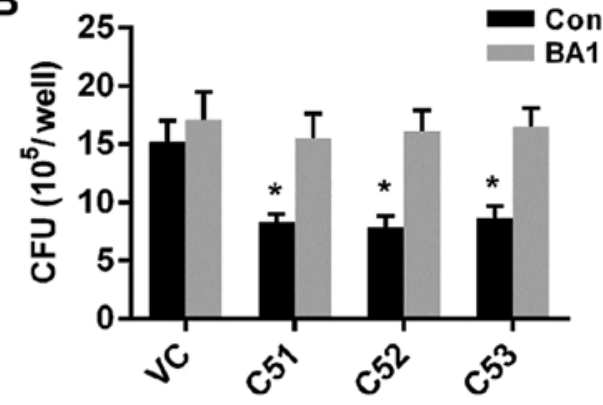

C

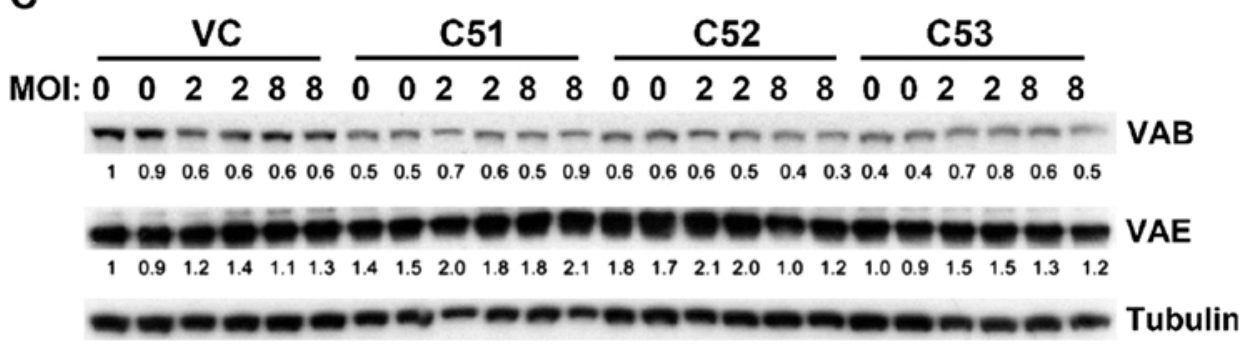

Figure 7. Effects of collagen $\alpha-5$ (IV) chain (Col4a5) knockdown on the V-ATPase activity and subunit expression in Raw264.7 macrophages. (A) Relative microsomal V-ATPase activity in control (VC) and Col4a5-knockdown (C51, C52 and C53) macrophage cell lines. (B) Colony forming units (CFUs) of mycobacterium bovis Calmette-Guérin (BCG) were counted in VC, C51, C52 and C53 cell lines infected with BCG at MOI 3, which were treated with the V-ATPase-specific inhibitor bafilomycin A1 (BA1) or in controls (Con). (C) Western blot analysis detected protein bands of the V-ATPase subunits B (VAB) and $\mathrm{E}$ (VAE) in VC, C51, C52 and C53 cell lines without BCG infection (MOI 0) or with $B C G$ infection (MOI 2,8). Tubulin served as loading control. ${ }^{\mathrm{P}<0.05}$ compared with VC.

by lentivirus-based antisense RNA methods, is the first one (to the best of our knowledge) to identify host genes that regulate mycobacteria via a macrophage-survival-based strategy. The death modality of MTB-infected macrophages plays different roles in the pathogenesis of tuberculosis and has extensive impacts on the outcome of the disease (35). Virulent MTB triggers necrosis and inhibits the apoptosis of host macrophages to evade host defense by affecting eicosanoid biosynthesis, while the attenuation of MTB induces apoptosis which then decreases bacterial viability (36). Controversy exists as to whether macrophage apoptosis is a self-protective mechanism of the host or simply a consequence of bacterial infection (37). Some investigators believe that host cell apoptosis deprives the pathogen of a protected intracellular environment, while others argue that MTB triggers macrophage apoptosis in order to escape the toxic intracellular environment and replicate in the extracellular space (37). In any case, the survival of virulent MTB-infected macrophages in vitro provides a powerful selective tool for the identification of macrophage mutants unable to take up MTB or to allow the bacterium to replicate. Our results suggest that under the conditions we used in this study, increased macrophage viability is consistent with increased control of the intracellular mycobacterial load. The 26 host genes we identified in the present study are different from those previously discovered, indicating that these host genes may be condition-dependent, infection phase-dependent, and/or specific for the macrophage survival-based strategy. Thus, it is not surprising that Col4a5 was not previously identified as a host gene reducing the MTB content in RNAi screens that targeted all kinases (34) or all genes in macrophage cell lines (19). However, in the present study, we demonstrated that Col4a5 deficiency had similar effect on the ability of macrophages to restrain the intracellular mycobacterial load in vitro. Although, Col4a5 deficiency enhanced two forms of anti-mycobacterial host defense in vitro, i.e., apoptosis and macrophage activation in the present study, it remains unclear whether it can lead to reduced MTB numbers in vivo. The pathogenesis of infectious disease is complex, and only limited aspects can be modeled in isolated cells. In general, the macrophage control of MTB in vitro appears to be far less effective than in the host. This presumably reflects the shortcomings of in vitro models, such as the exclusion of other cell types, differences in extracellular matrices, supra-physiological levels of oxygen and the use of heterologous sera (38).

Col4a5 encodes a $161 \mathrm{kDa}$ type IV collagen protein and its deficiency causes X-linked Alport syndrome, a hereditary nephritis (39). It is known that Col4a5 protein forms heterotrimers with other collagen IV subunits, i.e., Col4a3, Col4a4 or Col4a6 in basement membranes. The Col4a3/Col4a4/Col4a5 trimer is solid and rigid enough to enable the glomerular basement membrane to protect cells against harsh environments (39). Furthermore, although Col4a5 is highly expressed in the kidneys, lungs and inner ear, Col4a5-deficiency only causes diseases in the kidneys and ears, but not in the lungs (39). In this study, we describe a novel role of Col4a5 in negatively regulating V-ATPase activity in murine macrophages. V-ATPase is an essential cellular enzyme that acidifies intracellular vesicles, thus providing a mycobactericidal microenvironment (32). It is well known that mycobacteria attenuate phagosomal acidification, and initially this effect was proposed to be mainly mediated through the exclusion of V-ATPases from phagosomes, as a result of blocking of the fusion of 
phagosomes with V-ATPase-rich vesicles, including late endosomes and lysosomes (6). However, later studies indicated that phagosomes containing mycobacteria still acquire V-ATPases through interaction with early and recycling endosomes, which are also sources of V-ATPases (40). Currently, it is still unclear as to how Col4a5, an extracellular matrix protein, suppresses V-ATPase activity in intracellular vesicles, but does not alter its protein expression. There are at least two possible explanations. The first possibility is that it may not be full-length Col4a5, but internalized fragments that exert the inhibiting effects on cellular V-ATPase. This is consistent with our observation that Col4a 5 fragments may accumulate in macrophages after $2 \mathrm{~h}$ or longer incubation with mycobacteria (data not shown). Another possibility is that Col4a5 may interact with cell surface receptors that regulate the activity of V-ATPase-regulating protein kinases, which in turn regulate V-ATPase activity through phosphorylation of V-ATPase subunits (32).

There are several limitations to this study. Firstly, it is not certain to which degree the observed in vitro effects reflect the in vivo situation, particularly due to the fact that no additional information is available from Col4a5-knockout mice, which die shortly after birth (41). Therefore, a conditional knockout mouse model may help to elucidate the roles of Col4a5 in mycobacterial infection. Secondly, although not available yet, the screening and development of a specific Col4a5 inhibitor with high bioavailability and low off-target toxicity would be invaluable for further testing of Col4a5 functions in mouse models in vivo. Thirdly, it would be also interesting to identify the functional motifs in the Col4a5 protein, which are required for its effect on inhibiting mycobacterial infection in macrophages. Finally, in order to avoid the potential systematic toxicity, particularly the toxicity to the kidneys, Col4a5-targeted therapy should be limited to the lungs through pulmonary delivery. To this end, inhaled formulations of Col4a5-targeted therapy could be developed in the future.

In conclusion, we non-selectively inactivated genes in RAW264.7 macrophages, screened for cells that survived infection following virulent MTB infection, and identified 26 host genes, 9 of which were positive regulators of the expression of the Col4a5 gene, which encodes the dominant collagen IV subunit. In addition, we demonstrated that the knockdown of Col4a5 reduced mycobacterial viability and increased the survival of macrophages by promoting the acidification of intracellular vesicles, including endosomes, lysosomes and phagosomes. Finally, we demonstrated that Col4a5 knockdown may post-translationally increase microsomal vesicular ATPase activity in macrophages, leading to the acidification of both intracellular vesicles and culture media. Our findings reveal a promising role for Col4a5 in the regulation of murine macrophage responses to mycobacterial infection, identifying Col4a5 as a potential target for anti-mycobacterial therapy, and providing evidence of host-directed strategies that manipulate the survival of macrophages.

\section{Acknowledgements}

This study was supported by the Zhejiang Provincial Natural Science Foundation of China (grant no. LY14H010002) the Key Personnel Grant of Zhejiang Medicine and Health Platform (no. 2012RCA025), the Grant for Returned Overseas Chinese
Scholar of the Personnel Department of Zhejiang Province (no. J20120565), the Grant of Health and Family Planning Commission of Zhejiang province (no. 2009B060) and the 5050 project of Binjiang district.

\section{References}

1. BoseDasgupta $\mathrm{S}$ and Pieters J: Striking the right balance determines TB or not TB. Front Immunol 5: 455, 2014.

2. Zumla A, Raviglione M, Hafner R and von Reyn CF: Tuberculosis. N Engl J Med 368: 745-755, 2013.

3. Horsburgh CR Jr, Barry CE III and Lange C: Treatment of tuberculosis. N Engl J Med 373: 2149-2160, 2015.

4. Dey B and Bishai WR: Crosstalk between Mycobacterium tuberculosis and the host cell. Semin Immunol 26: 486-496, 2014.

5. Fu X, Ding M, Zhang N and Li J: Mycobacteriophages: An important tool for the diagnosis of Mycobacterium tuberculosis (Review). Mol Med Rep 12: 13-19, 2015.

6. Sturgill-Koszycki S, Schlesinger PH, Chakraborty P, Haddix PL, Collins HL, Fok AK, Allen RD, Gluck SL, Heuser J and Russell DG: Lack of acidification in Mycobacterium phagosomes produced by exclusion of the vesicular proton-ATPase. Science 263: 678-681, 1994.

7. Ferrari G, Langen H, Naito M and Pieters J: A coat protein on phagosomes involved in the intracellular survival of mycobacteria. Cell 97: 435-447, 1999.

8. Liu L, Liu J, Niu G, Xu Q and Chen Q: Mycobacterium tuberculosis 19-kDa lipoprotein induces Toll-like receptor 2-dependent peroxisome proliferator-activated receptor $\gamma$ expression and promotes inflammatory responses in human macrophages. Mol Med Rep 11: 2921-2926, 2015.

9. Brzostek A, Pawelczyk J, Rumijowska-Galewicz A, Dziadek B and Dziadek J: Mycobacterium tuberculosis is able to accumulate and utilize cholesterol. J Bacteriol 191: 6584-6591, 2009.

10. Fratti RA, Chua J and Deretic V: Induction of p38 mitogenactivated protein kinase reduces early endosome autoantigen 1 (EEA1) recruitment to phagosomal membranes. J Biol Chem 278: 46961-46967, 2003.

11. Lasunskaia EB, Campos MN, de Andrade MR, Damatta RA, Kipnis TL, Einicker-Lamas M and Da Silva WD: Mycobacteria directly induce cytoskeletal rearrangements for macrophage spreading and polarization through TLR2-dependent PI3K signaling. J Leukoc Biol 80: 1480-1490, 2006.

12. Vergne I, Chua J and Deretic V: Tuberculosis toxin blocking phagosome maturation inhibits a novel $\mathrm{Ca}^{2+} /$ calmodulin-PI3K hVPS34 cascade. J Exp Med 198: 653-659, 2003.

13. Mayer-Barber KD, Andrade BB, Oland SD, Amaral EP, Barber DL, Gonzales J, Derrick SC, Shi R, Kumar NP, Wei W, et al: Host-directed therapy of tuberculosis based on interleukin-1 and type I interferon crosstalk. Nature 511: 99-103, 2014.

14. Kindler V, Sappino AP, Grau GE, Piguet PF and Vassalli P: The inducing role of tumor necrosis factor in the development of bactericidal granulomas during BCG infection. Cell 56: 731-740, 1989.

15. Meyer CG and Thye T: Host genetic studies in adult pulmonary tuberculosis. Semin Immunol 26: 445-453, 2014.

16. Wallis RS and Hafner R: Advancing host-directed therapy for tuberculosis. Nat Rev Immunol 15: 255-263, 2015.

17. Zhao HF, L'Abbé D, Jolicoeur N, Wu M, Li Z, Yu Z and Shen SH: High-throughput screening of effective siRNAs from RNAi libraries delivered via bacterial invasion. Nat Methods 2: 967-973, 2005.

18. Chen R, Liliental JE, Kowalski PE, Lu Q and Cohen SN: Regulation of transcription of hypoxia-inducible factor- $1 \alpha$ (HIF-1 $\alpha$ ) by heat shock factors HSF2 and HSF4. Oncogene 30: 2570-2580, 2011

19. Kumar D, Nath L, Kamal MA, Varshney A, Jain A, Singh S and Rao KV: Genome-wide analysis of the host intracellular network that regulates survival of Mycobacterium tuberculosis. Cell 140: 731-743, 2010.

20. Lu Q, Wei W, Kowalski PE, Chang AC and Cohen SN: EST-based genome-wide gene inactivation identifies ARAP3 as a host protein affecting cellular susceptibility to anthrax toxin. Proc Natl Acad Sci USA 101: 17246-17251, 2004.

21. Wu K, Koo J, Jiang X, Chen R, Cohen SN and Nathan C: Improved control of tuberculosis and activation of macrophages in mice lacking protein kinase R. PLoS One 7: e30512, 2012. 
22. Philips JA, Rubin EJ and Perrimon N: Drosophila RNAi screen reveals CD36 family member required for mycobacterial infection. Science 309: 1251-1253, 2005.

23. Bao Z, Guan S, Cheng C, Wu S, Wong SH, Kemeny DM, Leung BP and Wong WS: A novel antiinflammatory role for andrographolide in asthma via inhibition of the nuclear factorkappaB pathway. Am J Respir Crit Care Med 179: 657-665, 2009.

24. Botelho RJ, Hackam DJ, Schreiber AD and Grinstein S: Role of COPI in phagosome maturation. J Biol Chem 275: 15717-15727, 2000.

25. Jankowski A, Scott CC and Grinstein S: Determinants of the phagosomal $\mathrm{pH}$ in neutrophils. J Biol Chem 277: 6059-6066, 2002.

26. Hackam DJ, Rotstein OD, Zhang W, Gruenheid S, Gros P and Grinstein S: Host resistance to intracellular infection: Mutation of natural resistance-associated macrophage protein 1 (Nramp1) impairs phagosomal acidification. J Exp Med 188: 351-364, 1998.

27. Vivas JR, Regnault B, Michel V, Bussière FI, Avé P, Huerre M, Labigne A, D'Elios MM and Touati E: Interferon gammasignature transcript profiling and IL-23 upregulation in response to Helicobacter pylori infection. Int J Immunopathol Pharmacol 21: $515-526,2008$

28. Ehrt S, Schnappinger D, Bekiranov S, Drenkow J, Shi S, Gingeras TR, Gaasterland T, Schoolnik G and Nathan C: Reprogramming of the macrophage transcriptome in response to interferon-gamma and Mycobacterium tuberculosis: signaling roles of nitric oxide synthase-2 and phagocyte oxidase. J Exp Med 194: 1123-1140, 2001.

29. Weiss G and Schaible UE: Macrophage defense mechanisms against intracellular bacteria. Immunol Rev 264: 182-203, 2015.

30. Vandal OH, Pierini LM, Schnappinger D, Nathan CF and Ehrt S: A membrane protein preserves intrabacterial $\mathrm{pH}$ in intraphagosomal Mycobacterium tuberculosis. Nat Med 14: 849-854, 2008

31. Jayachandran R, BoseDasgupta $S$ and Pieters J: Surviving the macrophage: Tools and tricks employed by Mycobacterium tuberculosis. Curr Top Microbiol Immunol 374: 189-209, 2013.
32. Jefferies KC, Cipriano DJ and Forgac M: Function, structure and regulation of the vacuolar $\left(\mathrm{H}^{+}\right)$-ATPases. Arch Biochem Biophys 476: 33-42, 2008.

33. Nathan C: Fresh approaches to anti-infective therapies. Sci Transl Med 4: 140sr2, 2012.

34. Jayaswal S, Kamal MA, Dua R, Gupta S, Majumdar T, Das G, Kumar D and Rao KV: Identification of host-dependent survival factors for intracellular Mycobacterium tuberculosis through an siRNA screen. PLoS Pathog 6: e1000839, 2010.

35. Divangahi M, Behar SM and Remold H: Dying to live: How the death modality of the infected macrophage affects immunity to tuberculosis. Adv Exp Med Biol 783: 103-120, 2013.

36. Behar SM, Divangahi M and Remold HG: Evasion of innate immunity by Mycobacterium tuberculosis: Is death an exit strategy? Nat Rev Microbiol 8: 668-674, 2010.

37. Parandhaman DK and Narayanan S: Cell death paradigms in the pathogenesis of Mycobacterium tuberculosis infection. Front Cell Infect Microbiol 4: 31, 2014.

38. Vogt $G$ and Nathan $C$ : In vitro differentiation of human macrophages with enhanced antimycobacterial activity. J Clin Invest 121: 3889-3901, 2011.

39. Hudson BG, Tryggvason K, Sundaramoorthy M and Neilson EG: Alport's syndrome, Goodpasture's syndrome, and type IV collagen. N Engl J Med 348: 2543-2556, 2003.

40. Medina E and North RJ: Evidence inconsistent with a role for the Bcg gene (Nramp1) in resistance of mice to infection with virulent Mycobacterium tuberculosis. J Exp Med 183: 1045$1051,1996$.

41. Rheault MN, Kren SM, Thielen BK, Mesa HA, Crosson JT, Thomas W, Sado Y, Kashtan CE and Segal Y: Mouse model of X-linked Alport syndrome. J Am Soc Nephrol 15: 1466-1474, 2004. 